\title{
Acaricidal properties of 5-methylfurfural identified from Valeriana fauriei and its structural analogues against synanthropic mites and Asian longhorned tick with color alterations
}

\author{
Seon-A Choi ${ }^{1}$, Jun-Hwan Park ${ }^{1}$,Hoi-Seon Lee ${ }^{2^{*}}$ and Ji-Hoon Lee ${ }^{1,3^{*}}$ (1)
}

\begin{abstract}
Acaricidal activities and color alterations of 5-methylfurfural derived from Valeriana fauriei essential oil and its structural analogues against Dermatophagoides farinae, D. pteronyssinus, Haemaphysalis longicornis and Tyrophagus putrescentiae were evaluated in the present study. Based on the $\mathrm{LD}_{50}$ values of 5-methylfurfural and its analogues, 4,5-dimethylfurfural showed the highest acaricidal activity $\left(\operatorname{LD}_{50} ; 9.95,9.91\right.$, and $\left.7.12 \mu \mathrm{g} / \mathrm{cm}^{2}\right)$, followed by 5 -methylfurfural $\left(11.87,11.00\right.$, and $\left.8.59 \mu \mathrm{g} / \mathrm{cm}^{2}\right)$, furfural $\left(12.94,13.25\right.$, and $\left.10.36 \mu \mathrm{g} / \mathrm{cm}^{2}\right)$, and $V$. fauriei essential oil (15.15, 13.64 , and $10.14 \mu \mathrm{g} / \mathrm{cm}^{2}$ ) against D. farinae, D. pteronyssinus and T. putrescentiae, respectively. However, all tested compounds did not show the acaricidal activities against $H$. longicornis. Interestingly, the color alterations of the mites and ticks were observed by furfural, 5-methylfurfural, and 4,5-dimethylfurfural from colorless to red brown during the acaricidal experiments. Furthermore, 4,5-dimethylfurfural which exhibited the highest acaricidal activity was formulated as nanoemulsion. The nanoemulsion of 4,5-dimethylfurfural showed higher acaricidal activity than it was emulsified in ethanol. The nanoemulsion was also found to show color changes of the mites and ticks from colorless to red brown. The results suggest that 5-methylfurfural and its analogues could be developed as an effective and easy-to-recognize acaricides to mites and ticks.
\end{abstract}

Keywords: Acaricidal activity, 5-methylfurfural, Mite color alteration, Dermatophagoides spp., Tyrophagus putrescentiae

\section{Introduction}

Global warming has influenced human health in many ways by accelerating the spread of various infectious agents. Especially, climate change has caused the growth of the arthropods to act as the allergens or pathogen vectors that occur the serious human health problems. The house dust mites, Dermatophagoides

\footnotetext{
*Correspondence: hoiseon@gmail.com; jhlee2@jbnu.ac.kr

1 Department of Bioenvironmental Chemistry, College of Agriculture and Life Sciences, Jeonbuk National University, Jeonju 54896, Republic of Korea

2 Biomedical Research Team, HS Biotech \& Holdings (HSBH) for Medical Research, Basel, Switzerland

Full list of author information is available at the end of the article
}

farinae and D. ptronyssinus (Acari: Pyroglyphidae) have been known to be the major source of potent allergens in house, which contribute to a number of allergic diseases such as asthma, eczema, and allergic rhinitis [1]. The storage mite, Tyrophagus putresceantiae (Acari: Acaridae) is found in stored products and induces allergic diseases with workers engaged in agriculture and food industries [2]. Furthermore, storage mite often spreads toxic bacteria and fungi such as Aspergillus spp. and Penicillium spp., because they can survive and grow by feeding on some seed-borne fungi [3]. The Asian longhorned tick, Haemaphysalis longicornis is an obligate blood-sucking ectoparasite and feed on a wide variety of vertebrate hosts [4], and is well known 
to carry the severe fever with thrombocytopenia syndrome (SFTS), including fever, gastrointestinal symptoms, thrombocytopenia, and leukocytopenia $[5,6]$. In these reasons, the arthropods have been the cause of decreasing the quality of human life and health.

One of the conventional control methods against mites and ticks is use of synthetic acaricides, such as avermectin, benzyl benzoate, chloropyrifos-methyl, DEET, etc. Although the synthetic acaricides are effective for the control of mites and ticks, long-term and excessive uses of synthetic acaricides can lead to adverse effects, such as resistance to acaricides, poor sustainability of the chemicals, environmental contamination, and side effects in non-target organisms [7]. Therefore, development of more efficient and safer alternatives for the control of mites and ticks is needed.

Recently, many studies focused on the plant extracts as new acaricidal products because they are biodegradable to non-toxic products and less likely to exhibit resistance [8]. Plant extracts are considered as the secondary metabolites that are not directly involved in the growth, development, or reproduction of the plant [9]. These constituents of plant extracts have already been described by many researches suggesting that they have bactericidal, antifungal, repellent, antiparasitic, and insecticidal properties [8]. In China, Valeriana fauriei has been used for the traditional medicine in the treatment of anxiety, insomnia, and sedation [10]. Several researchers have reported that the essential oils derived from Valeriana spp. exhibited the insecticidal activities against mosquitos including Aedes aegypti, A. albopictus, Anopheles stephensi, A. culicifacies, and Culex quinquefasciatus [11], and a nematode Bursaphelenchus xylophilus [12].

Although botanical insecticides are effective, their high molecular reactivity can be a limitation for manufacturing botanical insecticides. To maximize their biological properties, the essential oil and its active constituents need to be changed as the more stable forms. Nanoemulsions have stable colloidal properties with droplet sizes of $20-200 \mathrm{~nm}$, and which contain oil in their outer shell layer and a core portion inside active substances [13]. Because of their high kinetic stability, low viscosity, and optical transparency, nanoemulsions are used for many industrial applications including pharmaceutical, cosmetic, agrochemical, and polymerization fields [13].

In this study, we evaluated the acaricidal activities of $V$. fauriei essential oil and its constituents against $D$. farinae, D. pteronyssinus, H. longicornis, and T. putresceantiae and the color alterations of synanthropic mites. Furthermore, the acaricidal efficiency of the nanoemulsified plant oil components was evaluated using spray method to develop the new strategic acaricides.

\section{Materials and methods}

\section{Chemicals}

Amitraz (97\%) and $N, N$-diethyl- $m$-toluamide (DEET) (98\%) were purchased from TCI (Tokyo, Japan). Benzyl benzoate (99\%), 4,5-dimethylfurfural (97\%), furfural (99\%), furan (99\%), $\alpha$-guaiene (95\%), (-)- $\alpha$-gurjunene (97\%), 5-hydroxymethylfurfural (97\%), isovalerate (99\%), 2-methylfuran (99\%), 5-methylfurfural (99\%), methyl 3-methylvalerate (97\%), (-)-patchouli alcohol (98\%), and viridiflorol $(95 \%)$ were purchased from Sigma-Aldrich (St. Louis, MO, USA).

\section{Mite culture and tick collection}

The stock cultures of $D$. farinae, D. pteronyssinus, and $T$. putrescentiae were separately maintained without exposure to any acaricides over a period of 10 years [14]. Mites were maintained on fry feed no. 1 (Korea Special Feed Meal Co. Ltd., Jeonju, Korea) and dried yeast (1:1 by weight) and reared in the plastic box $(15 \times 12 \times 6 \mathrm{~cm})$ containing $28 \mathrm{~g}$ of a sterilized diet. The rearing cage was kept in temperature-controlled incubators at $26 \pm 1{ }^{\circ} \mathrm{C}$ and $70 \pm 5 \%$ relative humidity in continuous darkness within a plastic container $(20 \times 20 \times 20 \mathrm{~cm})$ that contained a supersaturated $\mathrm{NaCl}$ solution to prevent the escape of mites and to maintain relative humidity. Haemaphysalis longicornis nymphs were collected at Seogwipo city, Jeju Island, South Korea.

\section{Sample preparation and steam distillation extraction (SDE)} The roots of Valeriana fauriei $(3 \mathrm{~kg}$ ) were supplied from an herbal market in Jeonju, South Korea. The samples were washed three times with distilled water and dried in an oven at $38{ }^{\circ} \mathrm{C}$. After $6 \mathrm{~h}$, the dried samples finely homogenized with a grinder. Hexane was used as the extraction solvent. The $V$. fauriei root was diluted with distilled water in a flask and heated with $4 \mathrm{~h}$. After the extraction finished, anhydrous sodium sulfate was added to the extract to remove remain water. The residual solvent of the extract was removed with a rotary evaporator (EYELA auto jack NAJ-100, Tokyo, Japan) at $35{ }^{\circ} \mathrm{C}$ and stored at $4{ }^{\circ} \mathrm{C}$.

\section{Solvent extraction (SE)}

The powdered sample was placed in a $5000 \mathrm{~mL}$ glass flask with hexane $(1500 \mathrm{~mL})$. The extraction was performed on a shaking incubator (Edun, Seongnam, South Korea) at $230 \mathrm{rpm}$ and $26{ }^{\circ} \mathrm{C}$ for $48 \mathrm{~h}$. The extract was filtered to remove residues, and the residual solvent was removed with a rotary evaporator at $35^{\circ} \mathrm{C}$ and stored at $4{ }^{\circ} \mathrm{C}$.

\section{Supercritical fluid extraction (SFE)}

SFE was carried out as described by Lee et al. [15]. A supercritical fluid extractor (SCFE-0500, Ilshin autoclave, 
Daejeon, Korea) was used to obtain the extract of $V$. fauriei using supercritical $\mathrm{CO}_{2}$ at a temperature of $40-50{ }^{\circ} \mathrm{C}$ and a pressure of $40-400$ bar for $2 \mathrm{~h}$. A flow rate of supercritical $\mathrm{CO}_{2}$ was approximately $60 \mathrm{~mL} / \mathrm{min}$.

\section{Gas chromatography-mass spectrometry (GC-MS)}

The $V$. fauriei essential oils obtained by SDE, SE, and SFE were analyzed on Agilent-6890 GC system with Agilent-5973 MS operating in electron ionization mode at $70 \mathrm{eV}$. The system was equipped with a DB-5 fused silica capillary column $(30 \mathrm{~m} \times 0.25 \mathrm{~mm}$ i.d., J\&W Scientific, Folsom, CA, USA). The injection temperature was set at $50{ }^{\circ} \mathrm{C}$, which was gradually increased to $210{ }^{\circ} \mathrm{C}$ at $2{ }^{\circ} \mathrm{C} / \mathrm{min}$. Helium was used as carrier gas at constant flow rate of $0.8 \mathrm{ml} / \mathrm{min}$. The ion source temperature was $230{ }^{\circ} \mathrm{C}$. Mass spectra $(\mathrm{m} / \mathrm{z})$ were set to scan a range of 20-400 amu. The constituents of three $V$. fauriei essential oils were identified by comparing with standard compounds and mass spectra library [16].

\section{Preparation of nanoemulsion}

The oil-in-water nanoemulsion was prepared by using high-energy method and modified by the method of Sugumar et al. [17]. Briefly, emulsification was formulated by adding an organic phase to an aqueous phase. 4,5-Dimethylfurfural was mixed with Tween 80 (nonionic surfactant) in ratio of $1: 2(\mathrm{v} / \mathrm{v})$ as an organic phase, and glycerol $(5 \%, \mathrm{v} / \mathrm{v})$ was added to distilled water as aqueous phase. A coarse emulsion was prepared by blending an organic and aqueous phase using a magnetic stirrer (400 rpm) for $20 \mathrm{~min}$ at $40{ }^{\circ} \mathrm{C}$. Nanoemulsion was formed by passing the coarse emulsion through a $20 \mathrm{kHz}$ ultrasonic processor (VCX750, Sonics, USA) with a power output of $750 \mathrm{~W}$.

\section{Nanoemulsion characterization}

Particle size as polydispersity index (PDI) of the nanoemulsion were determined by a zeta potential and particle size analyzer (ELSZ-2000, Otsuka Electronics Co., Ltd., Osaka, Japan) at $25^{\circ} \mathrm{C}$. Sample was dispersed in distilled water in a ratio of 1:10. All results were obtained by measuring in triplicate and expressed as the mean \pm standard deviation.

\section{Contact + fumigant filter paper bioassay}

Contact + fumigant filter paper bioassay was used to measure the lethality and color alteration to $D$. farinae, D. pteronyssinus, and T. putrescentiae, which was modified to the description by Park and Lee [14]. Concentrations of each constituent in descending order $(110,55$, $27.5,13.75,6.88$, and $\left.3.44 \mu \mathrm{g} / \mathrm{cm}^{2}\right)$ were applied to $3.5 \mathrm{~cm}$ diameter filter paper (Whatman No. 1, Maidstone, UK). The filter paper was dried at room temperature. After
10 min, each filter paper was placed in the bottom of a petri dish $(3.5 \mathrm{~cm}$ i.d. $\times 1.0 \mathrm{~cm}$ deep; SPL life science, Pocheon, South Korea). Each group of 25-30 D. farinae, D. pteronyssinus, and T. putersceantiae was separately placed in the petri dish and covered with a lid, which wrapped with Bemis Parafilm M (Neenah, WI). The systems were kept in darkness at $27 \pm 1{ }^{\circ} \mathrm{C}$ and $78 \%$ relative humidity at $24 \mathrm{~h}$. Benzyl benzoate and DEET were used as positive control. Negative control consisted of $50 \mu \mathrm{L}$ of ethanol only. The color alteration of $D$. farinae, D. pteronyssinus, and T. putrescentiae were determined using a contact + fumigant filter paper bioassay and visualized by optical microscopy ( $\times 100$; Olympus, Japan). All treatments were replicated 5 times.

\section{Packet bioassay}

The packet bioassay was applied to measure the lethality of $V$. fauriei essential oil and its constituents against $H$. longicornis, which was modified from the description of Godara et al. [18]. Six formulations with decreasing concentrations $(110.00,55.00,27.50,13.75,6.88$, and $3.44 \mu \mathrm{g} /$ $\mathrm{cm}^{2}$ ) of each constituent dissolved in $200 \mu \mathrm{L}$ of ethanol were applied to $5.0 \times 10.0 \mathrm{~cm}$ filter paper. The filter paper was dried at room temperature at $10 \mathrm{~min}$. Dried each filter paper was folded in half and sealed with bulldog clips on both sides. The packet into which $20 \mathrm{H}$. longicornis larvae were dropped was sealed with a bulldog clip. The systems were kept in darkness at $27 \pm 1{ }^{\circ} \mathrm{C}$ and $80 \%$ relative humidity at $24 \mathrm{~h}$. Amitraz was used as positive control. Negative control consisted of $50 \mu \mathrm{L}$ of ethanol only. All treatments were replicated 5 times.

\section{Spray bioassay}

The acaricidal efficacy of 4,5-dimethylfurfural as emulsion dissolved in ethanol and nanoemulsion against $D$. farinae, D. pteronyssinus, and T. putrescentiae was measured by direct spray application method modified by Park and Lee [14]. Each group of 25-30 D. farinae, D. pteronyssinus, and T. putersceantiae was placed in the bottom of petri dish $(3.5 \mathrm{~cm}$ i.d. $\times 1.0 \mathrm{~cm}$ deep$)$. Each test sample was sprayed one time successively at $15 \mathrm{~cm}$ upwards onto the $3.5 \mathrm{~cm}$ diameter filter paper. After dried at $20 \mathrm{~min}$, each petri dish was covered with a lid and wrapped with Bemis Parafilm M. The systems were kept in darkness at $27 \pm 1{ }^{\circ} \mathrm{C}$ and $78 \%$ relative humidity at $24 \mathrm{~h}$. All treatments were replicated 5 times.

\section{Statistical analysis}

All data were corrected by Abbott's formula [19]. The 50 and $90 \%$ lethal dose $\left(\mathrm{LD}_{50}\right.$ and $\left.\mathrm{LD}_{90}\right)$ values were calculated by probit analysis. Relative toxicity (RT) was expressed based on the ratio of $\mathrm{LD}_{50}$ values of amitraz and DEET to $\mathrm{LD}_{50}$ values of each compound, 
as described previously [20]. The statistical difference between closed and open container bioassay was analyzed by Student's t test. SPSS version 12 software (SPSS Inc., Chicago, IL, USA) was used to analyze all data.

\section{Results \\ Acaricidal effects of $V$. fauriei essential oils obtained by SDE, SE, and SFE}

The mean yields of Valeriana fauriei essential oils obtained by steam distillation (SDE), solvent extraction (SE), and supercritical fluid extraction (SFE) were $0.67 \%$, $0.83 \%$, and $1.84 \%$, respectively. The acaricidal effects of $V$. fauriei essential oils obtained by SDE, SE, and SFE against $D$. farinae, D. pteronyssinus, $H$. longicornis, and T. putrescentiae were compared with the positive control (Table 1). Based on the $\mathrm{LD}_{50}$ values, the V. fauriei essential oil $\left(\mathrm{LD}_{50}, 15.15,13.64\right.$, and $\left.10.14 \mu \mathrm{g} / \mathrm{cm}^{2}\right)$ obtained by SDE was 1.28-1.83-fold more active than DEET (20.26, 17.40 , and $18.54 \mu \mathrm{g} / \mathrm{cm}^{2}$ ) against $D$. farinae, D. pteronyssinus, and $T$. putrescentiae, respectively. However, the essential oil obtained by SE and SFE did not show the acaricidal activities against $D$. farinae, D. pteronyssinus, and T. putrescentiae. The $V$. fauriei essential oil obtained by SDE, SE, and SFE also did not show the acaricidal activities against $H$. longicornis.

\section{GC-MS analysis of $V$. fauriei essential oils obtained by SDE, $\mathrm{SE}$, and SFE}

The components of $V$. fauriei essential oil obtained by SDE, SE, and SFE were investigated by GC-MS (Table 2). $V$. fauriei essential oil obtained by SDE yielded 17 compounds that represented $98.89 \%$ of the total oil, with viridiflorol (17.15\%), isovalerate (12.04\%), $\beta$-gurjunene (10.45\%), $\alpha$-guaiene (9.85\%), $\alpha$-patchoulene $(6.87 \%)$, methyl 3-methylvalerate (6.81\%), patchouli alcohol (6.44\%), $\alpha$-gurjunene (5.98\%), and 5-methylfurfural (5.41\%) being the major compounds. In V.fauriei essential oil obtained by SE, a total of 19 components were identified, which represented $100 \%$ of the total oil. The main compounds were 1-ethyl-4,4-dimethyl-2-cyclohexen1-ol (19.06\%), $\gamma$-sitosterol (18.80\%), baldrinal (11.36\%), patchouli alcohol (7.17\%), and 8-acetyl-5,5-dimethylbicyclo[2.2.2] octan-2-one (7.10\%). In the case of $V$. fauriei essential oil obtained by SFE, the main compounds were found to be 1-ethyl-4,4-dimethyl-2-cyclohexen-1-ol (15.77\%), $\gamma$-sitosterol (13.20\%), baldrinal (11.24\%), allyl valerate (10.08\%), 1-cyclohexyl-4,4-diethoxy-2-butyn1-one (6.86\%), 2,2-dicyclohexylmalononitrile (5.72\%), and S-propyl pentanethioate $(5.30 \%)$, which represented $97.77 \%$ of the total oil.

\section{Acaricidal effects of constituents of $V$. fauriei essential oil obtained by SDE}

The acaricidal effects of 7 commercial constituents [ $\alpha$-guaiene, $(-)$ - $\alpha$-gurjunene, isovalerate, 5 -methylfurfural, methyl 3-methylvalerate, (-)-patchouli alcohol, and viridiflorol] against $D$. farinae, D. pteronyssinus, $H$. longicornis, and T. putrescentiae were compared with positive control (Table 3). Based on the $\mathrm{LD}_{50}$ values, 5-methylfurfural $\left(11.87,11.00\right.$, and $\left.8.59 \mu \mathrm{g} / \mathrm{cm}^{2}\right)$ was 1.58-2.16-fold more active than DEET (20.26, 17.40, and $18.54 \mu \mathrm{g} / \mathrm{cm}^{2}$ ) against D. farinae, D. pteronyssinus, and $T$. putrescentiae, respectively, followed by isovalerate $\left(13.75,15.71\right.$, and $\left.11.23 \mu \mathrm{g} / \mathrm{cm}^{2}\right)$. However, isovalerate and 5-methylfurfural did not show the acaricidal activities against $H$. longicornis. $\alpha$-Guaiene, (-)- $\alpha$-gurjunene, methyl 3-methylvalerate, (-)-patchouli alcohol, and viridiflorol also did not show the acaricidal activities against $D$. farinae, D. pteronyssinus, $H$. longicornis, and $T$. putrescentiae.

\section{Acaricidal effects of 5-methylfurfural analogues}

To explore the structural relationships between 5-methylfurfural and the analogues for the acaricidal activities against $D$. farinae, $D$. pteronyssinus, $H$. longicornis, and $T$. putrescentiae, furan, 2-methylfuran, furfural, 4,5-dimethylfurfural, and 5-hydroxymethylfurfural were selected as 5-methylfurfural analogues for the testing (Table 4). Based on the $\mathrm{LD}_{50}$ values, 4,5-dimethylfurfural showed the most toxic activities among the analogues against D. farinae, D. pteronyssinus, and T. putrescentiae (9.95, 9.91, and $7.12 \mu \mathrm{g} / \mathrm{cm}^{2}$ ), respectively, followed by furfural $\left(12.94,13.25\right.$, and $\left.10.36 \mu \mathrm{g} / \mathrm{cm}^{2}\right)$, 5-hydroxymethylfurfural $\left(53.82,48.71\right.$, and $\left.57.58 \mu \mathrm{g} / \mathrm{cm}^{2}\right)$ and 2-methylfuran $\left(58.84,57.02\right.$, and $\left.39.71 \mu \mathrm{g} / \mathrm{cm}^{2}\right)$. However, furan did not show the acaricidal activities against $D$. farinae, D. pteronyssinus, and T. putrescentiae. The all 5-methylfurfural analogues did not show the acaricidal activities against $H$. longicornis. The color alterations by 5 -methylfurfural and its analogues were evaluated using the contact + fumigant filter paper bioassay against $D$. farinae, $D$. pteronyssinus, and T. putrescentiae (Fig. 1). After $24 \mathrm{~h}$ of treatment, $D$. farinae, D. pteronyssinus, and T. putrescentiae treated with furfural, 5-methylfurfural, and 4,5-dimethylfurfural exhibited changes in body color to red brown. However, D. farinae, D. pteronyssinus, and T. putrescentiae treated with benzyl benzoate and DEET did not exhibit the color change. 
Table 1 Acaricidal activities of Valeriana fauriei essential oils obtained by SDE, SE and SFE against D. farinae, D. pteronyssinus, H. longicornis and T. putrescentiae

\begin{tabular}{|c|c|c|c|c|c|c|c|c|}
\hline Samples & Insects & Bioassays & $\mathrm{LD}_{50}(95 \% \mathrm{Cl})$ & $\mathrm{LD}_{90}(95 \% \mathrm{Cl})$ & Slope \pm SE & $\mathrm{X}^{2}(\mathrm{df}, p)$ & RTD & RTA \\
\hline \multirow[t]{4}{*}{ V. fauriei SDE oil } & D. farinae & $\begin{array}{l}\text { Contact + fumigant }(\mu \mathrm{g} / \\
\left.\mathrm{cm}^{2}\right)\end{array}$ & 15.15 (11.90-19.28) & $38.04(28.22-62.13)$ & $3.21 \pm 0.50$ & $3.072(4,0.546)$ & 1.3 & - \\
\hline & D. pteronyssinus & $\begin{array}{l}\text { Contact + fumigant }(\mu \mathrm{g} / \\
\left.\mathrm{cm}^{2}\right)\end{array}$ & $13.64(10.87-17.31)$ & $32.12(24.22-51.32)$ & $3.47 \pm 0.56$ & $1.624(4,0.824)$ & 1.3 & - \\
\hline & H. longicornis & Packet (mg/ml) & $>50.00$ & - & - & - & - & - \\
\hline & T. putrescentiae & $\begin{array}{l}\text { Contact + fumigant }(\mu \mathrm{g} / \\
\left.\mathrm{cm}^{2}\right)\end{array}$ & $10.14(8.87-11.70)$ & $17.97(14.93-24.57)$ & $5.16 \pm 0.81$ & $2.232(4,0.693)$ & 1.8 & - \\
\hline \multirow[t]{4}{*}{ V. fauriei SE oil } & D. farinae & $\begin{array}{l}\text { Contact + fumigant }(\mu \mathrm{g} / \\
\left.\mathrm{cm}^{2}\right)\end{array}$ & $>110.00$ & - & - & - & - & - \\
\hline & D. pteronyssinus & $\begin{array}{l}\text { Contact + fumigant }(\mu \mathrm{g} / \\
\left.\mathrm{cm}^{2}\right)\end{array}$ & $>110.00$ & - & - & - & - & - \\
\hline & H. longicornis & Packet (mg/ml) & $>50.00$ & - & - & - & - & - \\
\hline & T.putrescentiae & $\begin{array}{l}\text { Contact + fumigant }(\mu \mathrm{g} / \\
\left.\mathrm{cm}^{2}\right)\end{array}$ & $>110.00$ & - & - & - & - & - \\
\hline \multirow[t]{4}{*}{ V. fauriei SFE oil } & D. farinae & $\begin{array}{l}\text { Contact + fumigant }(\mu \mathrm{g} / \\
\left.\mathrm{cm}^{2}\right)\end{array}$ & $>110.00$ & - & - & - & - & - \\
\hline & D. pteronyssinus & $\begin{array}{l}\text { Contact + fumigant }(\mu \mathrm{g} / \\
\left.\mathrm{cm}^{2}\right)\end{array}$ & $>110.00$ & - & - & - & - & - \\
\hline & H. longicornis & Packet (mg/ml) & $>50.00$ & - & - & - & - & - \\
\hline & T.putrescentiae & $\begin{array}{l}\text { Contact + fumigant }(\mu \mathrm{g} / \\
\left.\mathrm{cm}^{2}\right)\end{array}$ & $>110.00$ & - & - & - & - & - \\
\hline \multirow[t]{4}{*}{ Amitraz } & D. farinae & $\begin{array}{l}\text { Contact + fumigant }(\mu \mathrm{g} / \\
\left.\mathrm{cm}^{2}\right)\end{array}$ & $--^{\mathrm{a}}$ & - & - & - & - & - \\
\hline & D. pteronyssinus & $\begin{array}{l}\text { Contact + fumigant }(\mu \mathrm{g} / \\
\left.\mathrm{cm}^{2}\right)\end{array}$ & - & - & - & - & - & - \\
\hline & H. longicornis & Packet (mg/ml) & $23.83(18.67-30.46)$ & $64.18(47.12-105.32)$ & $2.98 \pm 0.44$ & $3.379(4,0.497)$ & - & 1.0 \\
\hline & T.putrescentiae & $\begin{array}{l}\text { Contact + fumigant }(\mu \mathrm{g} / \\
\left.\mathrm{cm}^{2}\right)\end{array}$ & - & - & - & - & - & - \\
\hline \multirow[t]{4}{*}{ Benzyl benzoate } & D. farinae & $\begin{array}{l}\text { Contact + fumigant }(\mu \mathrm{g} / \\
\left.\mathrm{cm}^{2}\right)\end{array}$ & $12.11(10.49-14.03)$ & $22.94(18.86-31.76)$ & $4.62 \pm 0.71$ & $3.660(4,0.454)$ & 1.7 & - \\
\hline & D. pteronyssinus & $\begin{array}{l}\text { Contact + fumigant }(\mu \mathrm{g} / \\
\left.\mathrm{cm}^{2}\right)\end{array}$ & $11.09(9.62-12.77)$ & $20.45(17.01-27.64)$ & $4.83 \pm 0.73$ & $3.319(4,0.506)$ & 1.6 & - \\
\hline & H. longicornis & Packet (mg/ml) & - & - & - & - & - & - \\
\hline & T.putrescentiae & $\begin{array}{l}\text { Contact + fumigant }(\mu \mathrm{g} / \\
\left.\mathrm{cm}^{2}\right)\end{array}$ & $9.95(8.49-11.48)$ & $18.08(15.15-24.05)$ & $4.94 \pm 0.77$ & $1.016(4,0.907)$ & 1.9 & - \\
\hline \multirow[t]{4}{*}{ DEET } & D. farinae & $\begin{array}{l}\text { Contact + fumigant }(\mu \mathrm{g} / \\
\left.\mathrm{cm}^{2}\right)\end{array}$ & $20.26(17.12-24.49)$ & 44.14 (34.22-68.35) & $3.79 \pm 0.59$ & $2.401(4,0.662)$ & 1.0 & - \\
\hline & D. pteronyssinus & $\begin{array}{l}\text { Contact + fumigant }(\mu \mathrm{g} / \\
\left.\mathrm{cm}^{2}\right)\end{array}$ & $17.40(15.12-20.31)$ & $31.77(25.98-45.00)$ & $4.90 \pm 0.79$ & $1.301(4,0.861)$ & 1.0 & - \\
\hline & H. longicornis & Packet (mg/ml) & - & - & - & - & - & - \\
\hline & T.putrescentiae & $\begin{array}{l}\text { Contact + fumigant }(\mu \mathrm{g} / \\
\left.\mathrm{cm}^{2}\right)\end{array}$ & $18.54(16.01-21.85)$ & $35.24(28.39-51.39)$ & $4.59 \pm 0.74$ & $2.226(4,0.694)$ & 1.0 & - \\
\hline
\end{tabular}

RTD relative toxicity $=L D_{50}$ value of $D E E T / L D_{50}$ value of each compound

$R T A$ relative toxicity $=L_{50}$ value of amitraz/LD $D_{50}$ value of each compound

a - , not tested

\section{Characterization of 4,5-dimethylfurfural nanoemulsion}

Particle size as PDI of 4,5-dimethylfurfural nanoemulsion was given in Fig. 2. Mean droplet size and PDI analysis showed that nanoemulsion of 4,5-dimethylfurfural exhibited small mean diameter $(161.3 \pm 32.3 \mathrm{~nm})$ and low polydispersity $(0.137 \pm 0.031)$ which means narrow particle size distribution (PDI <0.2).

\section{Acaricidal effect and color alteration}

\section{of 4,5-dimethylfurfural nanoemulsion}

The acaricidal effect of 4,5-dimethylfurfural nanoemulsion was evaluated by spray bioassay and compared with its emulsion dissolved in ethanol (Table 5). Based on the $\mathrm{LD}_{50}$ values, the 4,5-dimethylfurfural nanoemulsion $\left(\mathrm{LD}_{50}, 7.24,6.87\right.$, and $\left.5.59 \mu \mathrm{g} / \mathrm{cm}^{2}\right)$ was $1.27-1.44$-fold 
Table 2 GC-MS analysis of Valeriana fauriei essential oils by SDE, SE and SFE

\begin{tabular}{|c|c|c|c|c|c|}
\hline \multirow[t]{2}{*}{ No. } & \multirow[t]{2}{*}{ Compounds } & \multirow[t]{2}{*}{$\mathbf{R I}$} & \multicolumn{3}{|c|}{ Relative amount (\%) } \\
\hline & & & Steam & Solvent & Supercritical \\
\hline 1 & Methyl 3-methylvalerate & 820 & 6.81 & - & - \\
\hline 2 & Isovalerate & 811 & 12.04 & - & - \\
\hline 3 & 5-methylfurfural & 920 & 5.41 & - & - \\
\hline 4 & Limonene & 1033 & $-^{\mathrm{a}}$ & 2.27 & - \\
\hline 5 & $\beta$-hydroxyisovaleric acid & 966 & - & - & 2.25 \\
\hline 6 & Bornyl acetate & 1277 & - & 1.21 & - \\
\hline 7 & Allyl valerate & 974 & - & - & 10.08 \\
\hline 8 & a-santalene & 1420 & 1.19 & - & - \\
\hline 9 & a-bergamotene & 1431 & 0.97 & - & - \\
\hline 10 & $\beta$-gurjunene & 1403 & 10.45 & - & - \\
\hline 11 & $\beta$-caryophyllene & 1494 & 4.66 & 3.71 & - \\
\hline 12 & Seychellene & 1460 & 4.75 & 4.66 & 2.08 \\
\hline 13 & a-gurjunene & 1419 & 5.98 & 2.87 & - \\
\hline 14 & $\beta$-selinene & 1436 & - & 1.04 & - \\
\hline 15 & a-patchoulene & 1456 & 6.87 & 2.94 & - \\
\hline 16 & $(+)$-cyclosativene & 1125 & 1.23 & - & - \\
\hline 17 & 2,8,8-trimethyl-4-methylene-2-vinylbicyclo[5.2.0]nonane & 1407 & 2.69 & - & - \\
\hline 18 & a-guaiene & 1490 & 9.85 & 1.12 & - \\
\hline 19 & a-panasinsene & 1416 & 1.52 & 1.60 & - \\
\hline 20 & Viridiflorol & 1754 & 17.15 & - & - \\
\hline 21 & $\beta$-eudesmol & 1593 & 0.88 & - & - \\
\hline 22 & Patchouli alcohol & 1659 & 6.44 & 7.17 & 4.82 \\
\hline 23 & Baldrinal & 1689 & - & 11.36 & 11.24 \\
\hline 24 & Palmitic acid & 1968 & - & - & 3.18 \\
\hline 25 & 1-ethyl-4,4-dimethyl-2-cyclohexen-1-ol & 1147 & - & 19.06 & 15.77 \\
\hline 26 & 3-Acetoxycinnamic acid & 1737 & - & 3.33 & - \\
\hline 27 & 6-hydroxy-5,7,8-trimethyl-2-chromanone & 1952 & - & - & 2.95 \\
\hline 28 & Methyl 3-acetoxy-3-hydroxy-2-methylpropanoate & 1150 & - & - & 1.68 \\
\hline 29 & 4-carvomenthenol & 2458 & - & - & 2.28 \\
\hline 30 & Artemisia ketone & 1042 & - & 1.59 & - \\
\hline 31 & 8-acetyl-5,5-dimethyl-bicyclo[2.2.2]octan-2-one & 1464 & - & 7.10 & - \\
\hline 32 & 1-cyclohexyl-4,4-diethoxy-2-butyn-1-one & 1717 & - & - & 6.86 \\
\hline 33 & 2,6-dimethyl-6-nitro-2-hepten-4-one & 1334 & - & 2.41 & - \\
\hline 34 & 2,2-dicyclohexylmalononitrile & 2045 & - & - & 5.72 \\
\hline 35 & S-propyl pentanethioate & 1139 & - & 2.85 & 5.30 \\
\hline 36 & (-)-praeruptorin B & 3097 & - & 4.91 & 2.30 \\
\hline 37 & 1,6-dibromohexane & 1210 & - & - & 2.14 \\
\hline 38 & 2,3,7-trimethyl-3-vinyl-oct-6-enoic acid & 1496 & - & - & 3.11 \\
\hline 39 & (+)-a-tocopherol & 3149 & - & - & 2.81 \\
\hline 40 & $\gamma$-sitosterol & 2731 & - & 18.80 & 13.20 \\
\hline \multicolumn{6}{|l|}{ Major grouped compounds } \\
\hline Acid & & & 12.04 & 6.18 & 13.84 \\
\hline Aldehydes & & & 5.41 & 11.36 & 11.24 \\
\hline Aliphatic ester & & & 6.81 & - & - \\
\hline Alkanes & & & 3.92 & - & - \\
\hline Ketones & & & - & 1.59 & - \\
\hline Monoterpene alcohol & & & - & 19.06 & 18.05 \\
\hline Monoterpene esters & & & - & 3.62 & 11.76 \\
\hline
\end{tabular}


Table 2 (continued)

\begin{tabular}{|c|c|c|c|c|}
\hline \multirow[t]{2}{*}{ No. } & \multirow[t]{2}{*}{ RI } & \multicolumn{3}{|c|}{ Relative amount (\%) } \\
\hline & & Steam & Solvent & Supercritical \\
\hline \multicolumn{2}{|c|}{ Monoterpene ketone } & - & - & - \\
\hline \multicolumn{2}{|c|}{ Monoterpene hydrocarbons } & - & 2.27 & - \\
\hline \multicolumn{2}{|c|}{ Sesquiterpene alcohols } & 47.64 & 7.17 & 7.77 \\
\hline \multicolumn{2}{|c|}{ Sesquiterpene esters } & - & - & 6.86 \\
\hline \multicolumn{2}{|c|}{ Sesquiterpene ethers } & - & 7.10 & - \\
\hline \multicolumn{2}{|c|}{ Sesquiterpenene hydrocarbons } & 23.07 & 17.94 & 2.08 \\
\hline \multicolumn{2}{|c|}{ Sesquiterpenene nitrile } & - & - & 5.72 \\
\hline \multicolumn{2}{|c|}{ Triterpenoids } & - & 18.80 & 16.01 \\
\hline \multicolumn{2}{|c|}{ Pyranocoumarins } & - & 4.91 & 2.30 \\
\hline \multicolumn{2}{|c|}{ Total (\%) } & 98.89 & 100.00 & 97.77 \\
\hline \multicolumn{2}{|c|}{ Yield (\%) } & 0.66 & 0.84 & 2.21 \\
\hline
\end{tabular}

$R /$ retention index

${ }^{\text {a }}$ Not detected

more active than the emulsion dissolved in ethanol against $D$. farinae, D. pteronyssinus, and T. putrescentiae, respectively. The color alterations by 4,5 -dimethylfurfural nanoemulsion were evaluated using the spray bioassay (Fig. 3). After $24 \mathrm{~h}$ of treatment, D. farinae, D. pteronyssinus, and T. putrescentiae treated with the nanoemulsion exhibited changes in body color to red brown.

\section{Discussion}

In this study, the acaricidal activities of $V$. fauriei essential oil obtained by three extraction methods (SDE, SE, and SFE) and its constituents were found. The essential oil obtained by SDE affected the highest acaricidal activity among the tested oils against $D$. farinae, D. pteronyssinus, and T. putrescentiae, despite the lowest mean yield. Unfortunately, the oil did not show any toxic response against $H$. longicornis. The differential susceptibility of the essential oils to tested mites and ticks may be influenced by biological factors (body size, weight, external structure, etc.) and biochemical properties (detoxification enzyme activity such as esterases, cytochrome P450 monooxygenases, and glutathione-S-transferases). According to previous studies, the Coriandrum sativum essential oil obtained by SDE exhibited the higher acaricidal activities against $T$. putrescentiae $\left(\mathrm{LD}_{50} ; 19.29 \mu \mathrm{g} /\right.$ $\mathrm{cm}^{2}$ ) than the essential oils obtained by SE and SFE [15]. In contrast, the Cinnamomum cassia essential oil obtained by SE revealed the higher acaricidal activities against $T$. putrescentiae $\left(\mathrm{LD}_{50} ; 2.60 \mu \mathrm{g} / \mathrm{cm}^{2}\right)$ than the essential oils obtained by SDE and SFE [21]. These results indicated that the acaricidal activity varied with the extraction methods of essential oil. To our knowledge, this study is the first report on the acaricidal activities of $V$. fauriei essential oil against D. farinae, D. pteronyssinus, and T. putrescentiae. It is suggested that $V$. fauriei essential oil obtained by SDE is potent for the development of effective and efficient natural acaricide for the control of the synanthropic mites.

The SDE is generally used for extraction to volatile compounds, especially monoterpenes, which exhibit the acaricidal activity [22]. However, the $V$. fauriei essential oils showed different results from those of previous studies. The most abundant component in the V. fauriei essential oils obtained by SDE was viridiflorol (17.15\%), which is one of sesquiterpene compounds. This essential oil exhibited higher sesquiterpene composition (70.71\%) than those obtained by the SE and SFE $(32.21 \%$ and $22.43 \%$, respectively). There are many reports on the abundance of sesquiterpenes in the essential oils of Valeriana spp. The most abundant fraction of the roots and rhizomes of $V$. officinalis was sesquiterpene fraction (70.5\%) and its main constituent was valerianol (57.3\%) [23]. In the essential oil of $V$. sisymbriifolia, b-atlantone and 14-hydroxy-9-epi-(E)-caryophyllene (16.3\%) were the main constituents and oxygenated sesquiterpenes (39.1\%) constituted the major portion [24]. These differences of the compositions may be caused by different cultivation conditions, such as climate, season, geographical environment, and growth stage of plants [25].

To explore the active compounds in $V$. fauriei essential oil obtained by SDE, the acaricidal potential of 7 commercial constituents (over 5\%) were evaluated by acaricidal experimentation. As a result, 5-methylfurfural and isovalerate had the highest lethality against $D$. farinae, D. pteronyssinus, and T. putrescentiae. 5 methylfurfural showed 1.7, 1.6 and 2.2 times more toxic than DEET, and isovalerate showed $1.5,1.1$ and 1.7 times more toxic than DEET against D. farinae, D. pteronyssinus, and $T$. 
Table 3 Acaricidal activities of 7 constituents of Valeriana fauriei essential oil obtained by SDE against D. farinae, D. pteronyssinus, H. longicornis and T. putrescentiae

\begin{tabular}{|c|c|c|c|c|c|c|c|c|}
\hline Compound & Insect & Bioassay & $\mathrm{LD}_{50}(95 \% \mathrm{Cl})$ & $\mathrm{LD}_{90}(95 \% \mathrm{Cl})$ & Slope \pm SE & $\mathrm{x}^{2}(\mathrm{df}, p)$ & RTD & $\overline{\text { RTA }}$ \\
\hline \multirow[t]{4}{*}{$\begin{array}{l}\text { Methyl 3-methylvaler- } \\
\text { ate }\end{array}$} & D. farinae & $\begin{array}{l}\text { Contact }+ \text { fumigant } \\
\left(\mu \mathrm{g} / \mathrm{cm}^{2}\right)\end{array}$ & $>110.00$ & - & - & - & - & - \\
\hline & D. pteronyssinus & $\begin{array}{l}\text { Contact }+ \text { fumigant } \\
\left(\mu \mathrm{g} / \mathrm{cm}^{2}\right)\end{array}$ & $>110.00$ & - & - & - & - & - \\
\hline & H. longicornis & Packet $(\mathrm{mg} / \mathrm{ml})$ & $>50.00$ & - & - & - & - & - \\
\hline & T.putrescentiae & $\begin{array}{l}\text { Contact }+ \text { fumigant } \\
\left(\mu \mathrm{g} / \mathrm{cm}^{2}\right)\end{array}$ & $>110.00$ & - & - & - & - & - \\
\hline \multirow[t]{4}{*}{ Isovalerate } & D. farinae & $\begin{array}{l}\text { Contact }+ \text { fumigant } \\
\left(\mu \mathrm{g} / \mathrm{cm}^{2}\right)\end{array}$ & 13.75 (10.68-17.58) & $36.30(26.72-60.05)$ & $3.04 \pm 0.48$ & $4.261(4,0.372)$ & 1.5 & - \\
\hline & D. pteronyssinus & $\begin{array}{l}\text { Contact }+ \text { fumigant } \\
\left(\mu \mathrm{g} / \mathrm{cm}^{2}\right)\end{array}$ & $15.71(12.42-19.87)$ & $37.55(28.15-60.44)$ & $3.39 \pm 0.54$ & $2.430(4,0.657)$ & 1.1 & - \\
\hline & H. longicornis & Packet (mg/ml) & $>50.00$ & - & - & - & - & - \\
\hline & T.putrescentiae & $\begin{array}{l}\text { Contact }+ \text { fumigant } \\
\left(\mu \mathrm{g} / \mathrm{cm}^{2}\right)\end{array}$ & $11.23(8.76-14.23)$ & $27.74(20.73-45.08)$ & $3.27 \pm 0.53$ & $2.990(4,0.657)$ & 1.7 & - \\
\hline \multirow[t]{4}{*}{ 5-methylfurfural } & D. farinae & $\begin{array}{l}\text { Contact }+ \text { fumigant } \\
\left(\mu \mathrm{g} / \mathrm{cm}^{2}\right)\end{array}$ & $11.87(9.75-14.56)$ & $27.64(21.06-44.36)$ & $3.49 \pm 0.57$ & $1.669(4,0.796)$ & 1.7 & - \\
\hline & D. pteronyssinus & $\begin{array}{l}\text { Contact }+ \text { fumigant } \\
\left(\mu \mathrm{g} / \mathrm{cm}^{2}\right)\end{array}$ & $11.00(9.05-13.41)$ & $24.99(19.21-39.71)$ & $3.60 \pm 0.60$ & $1.729(4,0.785)$ & 1.6 & - \\
\hline & H. longicornis & Packet (mg/ml) & $>50.00$ & - & - & - & - & - \\
\hline & T.putrescentiae & $\begin{array}{l}\text { Contact }+ \text { fumigant } \\
\left(\mu \mathrm{g} / \mathrm{cm}^{2}\right)\end{array}$ & $8.59(6.47-11.28)$ & 28.07 (19.77-49.41) & $2.49 \pm 0.38$ & $5.047(4,0.283)$ & 2.2 & - \\
\hline \multirow[t]{4}{*}{ (-)-a-gurjunene } & D. farinae & $\begin{array}{l}\text { Contact }+ \text { fumigant } \\
\left(\mu \mathrm{g} / \mathrm{cm}^{2}\right)\end{array}$ & $>110.00$ & - & - & - & - & - \\
\hline & D. pteronyssinus & $\begin{array}{l}\text { Contact }+ \text { fumigant } \\
\left(\mu \mathrm{g} / \mathrm{cm}^{2}\right)\end{array}$ & $>110.00$ & - & - & - & - & - \\
\hline & H. longicornis & Packet (mg/ml) & $>50.00$ & - & - & - & - & - \\
\hline & T.putrescentiae & $\begin{array}{l}\text { Contact }+ \text { fumigant } \\
\left(\mu \mathrm{g} / \mathrm{cm}^{2}\right)\end{array}$ & $>110.00$ & - & - & - & - & - \\
\hline \multirow[t]{4}{*}{ a-guaiene } & D. farinae & $\begin{array}{l}\text { Contact }+ \text { fumigant } \\
\left(\mu \mathrm{g} / \mathrm{cm}^{2}\right)\end{array}$ & $>110.00$ & - & - & - & - & - \\
\hline & D. pteronyssinus & $\begin{array}{l}\text { Contact }+ \text { fumigant } \\
\left(\mu \mathrm{g} / \mathrm{cm}^{2}\right)\end{array}$ & $>110.00$ & - & - & - & - & - \\
\hline & H. longicornis & Packet (mg/ml) & $>50.00$ & - & - & - & - & - \\
\hline & T.putrescentiae & $\begin{array}{l}\text { Contact }+ \text { fumigant } \\
\left(\mu \mathrm{g} / \mathrm{cm}^{2}\right)\end{array}$ & $>110.00$ & - & - & - & - & - \\
\hline \multirow[t]{4}{*}{ Viridiflorol } & D. farinae & $\begin{array}{l}\text { Contact }+ \text { fumigant } \\
\left(\mu \mathrm{g} / \mathrm{cm}^{2}\right)\end{array}$ & $>110.00$ & - & - & - & - & - \\
\hline & D. pteronyssinus & $\begin{array}{l}\text { Contact }+ \text { fumigant } \\
\left(\mu \mathrm{g} / \mathrm{cm}^{2}\right)\end{array}$ & $>110.00$ & - & - & - & - & - \\
\hline & H. longicornis & Packet (mg/ml) & $>50.00$ & - & - & - & - & - \\
\hline & T.putrescentiae & $\begin{array}{l}\text { Contact }+ \text { fumigant } \\
\left(\mu \mathrm{g} / \mathrm{cm}^{2}\right)\end{array}$ & $>110.00$ & - & - & - & - & - \\
\hline \multirow[t]{4}{*}{ (-)-patchouli alcohol } & D. farinae & $\begin{array}{l}\text { Contact }+ \text { fumigant } \\
\left(\mu \mathrm{g} / \mathrm{cm}^{2}\right)\end{array}$ & $>110.00$ & - & - & - & - & - \\
\hline & D. pteronyssinus & $\begin{array}{l}\text { Contact }+ \text { fumigant } \\
\left(\mu \mathrm{g} / \mathrm{cm}^{2}\right)\end{array}$ & $>110.00$ & - & - & - & - & - \\
\hline & H. longicornis & Packet (mg/ml) & $>50.00$ & - & - & - & - & - \\
\hline & T.putrescentiae & $\begin{array}{l}\text { Contact + fumigant } \\
\left(\mu \mathrm{g} / \mathrm{cm}^{2}\right)\end{array}$ & $>110.00$ & - & - & - & - & - \\
\hline
\end{tabular}


Table 3 (continued)

\begin{tabular}{|c|c|c|c|c|c|c|c|c|}
\hline Compound & Insect & Bioassay & $\mathrm{LD}_{50}(95 \% \mathrm{Cl})$ & $\mathrm{LD}_{90}(95 \% \mathrm{Cl})$ & Slope \pm SE & $x^{2}(d f, p)$ & RTD & RTA \\
\hline \multirow[t]{4}{*}{ Amitraz } & D. farinae & $\begin{array}{l}\text { Contact }+ \text { fumigant } \\
\left(\mu \mathrm{g} / \mathrm{cm}^{2}\right)\end{array}$ & $-^{\mathrm{a}}$ & - & - & - & - & - \\
\hline & D. pteronyssinus & $\begin{array}{l}\text { Contact }+ \text { fumigant } \\
\left(\mu \mathrm{g} / \mathrm{cm}^{2}\right)\end{array}$ & - & - & - & - & - & - \\
\hline & H. longicornis & Packet (mg/ml) & $23.83(18.67-30.46)$ & 64.18 (47.12-105.32) & $2.98 \pm 0.44$ & $3.379(4,0.497)$ & - & 1.0 \\
\hline & T. putrescentiae & $\begin{array}{l}\text { Contact }+ \text { fumigant } \\
\left(\mu \mathrm{g} / \mathrm{cm}^{2}\right)\end{array}$ & - & - & - & - & - & - \\
\hline \multirow[t]{4}{*}{ Benzyl benzoate } & D. farinae & $\begin{array}{l}\text { Contact }+ \text { fumigant } \\
\left(\mu \mathrm{g} / \mathrm{cm}^{2}\right)\end{array}$ & $12.11(10.49-14.03)$ & $22.94(18.86-31.76)$ & $4.62 \pm 0.71$ & $3.660(4,0.454)$ & 1.7 & - \\
\hline & D. pteronyssinus & $\begin{array}{l}\text { Contact }+ \text { fumigant } \\
\left(\mu \mathrm{g} / \mathrm{cm}^{2}\right)\end{array}$ & $11.09(9.62-12.77)$ & $20.45(17.01-27.64)$ & $4.83 \pm 0.73$ & $3.319(4,0.506)$ & 1.6 & - \\
\hline & H. longicornis & Packet (mg/ml) & - & - & - & - & - & - \\
\hline & T.putrescentiae & $\begin{array}{l}\text { Contact }+ \text { fumigant } \\
\left(\mu \mathrm{g} / \mathrm{cm}^{2}\right)\end{array}$ & $9.95(8.49-11.48)$ & 18.08 (15.15-24.05) & $4.94 \pm 0.77$ & $1.016(4,0.907)$ & 1.9 & - \\
\hline \multirow[t]{4}{*}{ DEET } & D. farinae & $\begin{array}{l}\text { Contact }+ \text { fumigant } \\
\left(\mu \mathrm{g} / \mathrm{cm}^{2}\right)\end{array}$ & $20.26(17.12-24.49)$ & $44.14(34.22-68.35)$ & $3.79 \pm 0.59$ & $2.401(4,0.662)$ & 1.0 & - \\
\hline & D. pteronyssinus & $\begin{array}{l}\text { Contact }+ \text { fumigant } \\
\left(\mu \mathrm{g} / \mathrm{cm}^{2}\right)\end{array}$ & $17.40(15.12-20.31)$ & $31.77(25.98-45.00)$ & $4.90 \pm 0.79$ & $1.301(4,0.861)$ & 1.0 & - \\
\hline & H. longicornis & Packet (mg/ml) & - & - & - & - & - & - \\
\hline & T.putrescentiae & $\begin{array}{l}\text { Contact }+ \text { fumigant } \\
\left(\mu \mathrm{g} / \mathrm{cm}^{2}\right)\end{array}$ & $18.54(16.01-21.85)$ & 35.24 (28.39-51.39) & $4.59 \pm 0.74$ & $2.226(4,0.694)$ & 1.0 & - \\
\hline
\end{tabular}

RTD relative toxicity $=L D_{50}$ value of $D E E T / L D_{50}$ value of each compound

$R T A$ Relative toxicity $=\mathrm{LD}_{50}$ value of amitraz/LD $\mathrm{D}_{50}$ value of each compound

${ }_{-}$, not tested

putrescentiae. On the other hand, viridiflorol that was the most abundant compound in the $V$. fauriei SDE oil showed no acaricidal activities in all tested concentrations. Although isovalerate and 5-methylfurfural did not account the high percentage in the essential oil, they have a potential as an alternative of synthetic acaricide because of their effectiveness. Liu et al. [26] reported that isovalerate derived from Valeriana jatamansi exhibited stronger lethal toxicity against Liposcelis bostrychophila with the $\mathrm{LC}_{50}$ value of $426.34 \mu \mathrm{g} / \mathrm{cm}^{2}$.

Many potential acaricides derived from plants have been explored for mite control. However, complete elimination of mite allergens (residual mite excrements, dead mites, and eggs) is actually impossible because of their invisible size. These problems led to the development of a new strategies for the control of synanthropic mite allergens. Interestingly, this study showed the color alteration of 5-methylfurfural against $D$. farinae, D. pteronyssinus, and T. putrescentiae (Fig. 1). The mechanism of the discoloration could be associated with melanization performed by polyphenol oxidase and tyrosinase. Polyphenol oxidase is the enzyme of the insect innate immune system by producing melanin, which prevents the introduction of outside substances [27]. Tyrosinase plays a role in protection of UV damage and initiates melanization by hydroxylating tyrosine to (dopa) and oxidizing the (dopa) to dopaquinone [28]. Similar to this study, plumbagin, naphthazarin, dichlone, 2-bromo-1,4-naphthoquinone [29], 2,3-dihydroxybenzaldehyde [30], and citral [14] were reported as color alteration agents. In this regard, the red brown color intensity was not correlated with the toxicity of treated components.

To evaluate the structure-activity relationships of 5-methylfurfural and its structural analogues, furan, 2-methylfuran, furfural, 4,5-dimethylfurfural, and 5-hydroxymethylfurfural were tested. The SAR was mainly based upon by bringing about difference of substituents on furan ring. In the contact + fumigant bioassay, the acaricidal activities of the structural analogues containing a methyl $\left(\mathrm{CH}_{3}\right)$ functional group (5-methylfurfural, 4,5-dimethylfurfural, and 2-methylfuran) were more toxic than those of hydroxymethyl functional group (5-hydroxymethylfurfural) or no functional group (furan). The color alterations of $D$. farinae, D. pteronyssinus, and T. putrescentiae were also observed when treated with the structural analogues containing a methyl functional group (5-methylfurfural and 4,5-dimethylfurfural) except for 2-methylfuran. Oh et al. [31] showed that acetophenone derivatives containing a methyl group exhibited strong acaricidal activity against $D$. farinae and D. pteronyssinus. Furthermore, Yang et al. [30] reported that the number of methyl functional groups of 
Table 4 Acaricidal activities of 5-methyhlfurfural analogues against D. farinae, D. pteronyssinus, H. longicornis and T. putrescentiae

\begin{tabular}{|c|c|c|c|c|c|c|c|c|}
\hline Compound & Insect & Bioassay & $\mathrm{LD}_{50}(95 \% \mathrm{Cl})$ & $\mathrm{LD}_{90}(95 \% \mathrm{Cl})$ & Slope \pm SE & $\mathrm{X}^{2}(\mathrm{df}, p)$ & RTD & RTA \\
\hline \multirow[t]{4}{*}{ Furan } & D. farinae & $\begin{array}{l}\text { Contact }+ \text { fumigant } \\
\left(\mu \mathrm{g} / \mathrm{cm}^{2}\right)\end{array}$ & $>110.00$ & - & - & - & - & - \\
\hline & D. pteronyssinus & $\begin{array}{l}\text { Contact }+ \text { fumigant } \\
\left(\mu \mathrm{g} / \mathrm{cm}^{2}\right)\end{array}$ & $>110.00$ & - & - & - & - & - \\
\hline & H. longicornis & Packet (mg/ml) & $>50.00$ & - & - & - & - & - \\
\hline & T.putrescentiae & $\begin{array}{l}\text { Contact }+ \text { fumigant } \\
\left(\mu \mathrm{g} / \mathrm{cm}^{2}\right)\end{array}$ & $>110.00$ & - & - & - & - & - \\
\hline \multirow[t]{4}{*}{ 2-methylfuran } & D. farinae & $\begin{array}{l}\text { Contact }+ \text { fumigant } \\
\left(\mu \mathrm{g} / \mathrm{cm}^{2}\right)\end{array}$ & $58.84(47.55-72.92)$ & $119.38(92.62-184.70)$ & $4.17 \pm 0.72$ & $0.404(4,0.982)$ & 0.3 & - \\
\hline & D. pteronyssinus & $\begin{array}{l}\text { Contact }+ \text { fumigant } \\
\left(\mu \mathrm{g} / \mathrm{cm}^{2}\right)\end{array}$ & $57.02(45.49-71.60)$ & $127.56(96.83-202.80)$ & $3.67 \pm 0.60$ & $1.179(4,0.882)$ & 0.3 & - \\
\hline & H. longicornis & Packet (mg/ml) & $>50.00$ & - & - & - & - & - \\
\hline & T. putrescentiae & $\begin{array}{l}\text { Contact }+ \text { fumigant } \\
\left(\mu \mathrm{g} / \mathrm{cm}^{2}\right)\end{array}$ & $39.71(31.96-49.61)$ & $84.53(64.73-133.05)$ & $3.91 \pm 0.65$ & $2.338(4,0.674)$ & 0.5 & - \\
\hline \multirow[t]{4}{*}{ Furfural } & D. farinae & $\begin{array}{l}\text { Contact }+ \text { fumigant } \\
\left(\mu \mathrm{g} / \mathrm{cm}^{2}\right)\end{array}$ & $12.94(10.50-15.88)$ & $24.95(19.65-37.72)$ & $4.49 \pm 0.79$ & $0.648(4,0.958)$ & 1.6 & - \\
\hline & D. pteronyssinus & $\begin{array}{l}\text { Contact }+ \text { fumigant } \\
\left(\mu \mathrm{g} / \mathrm{cm}^{2}\right)\end{array}$ & $13.25(10.70-16.42)$ & $26.96(20.92-41.66)$ & $4.15 \pm 0.71$ & $0.513(4,0.972)$ & 1.3 & - \\
\hline & H. longicornis & Packet (mg/ml) & $>50.00$ & - & - & - & - & - \\
\hline & T.putrescentiae & $\begin{array}{l}\text { Contact }+ \text { fumigant } \\
\left(\mu \mathrm{g} / \mathrm{cm}^{2}\right)\end{array}$ & $10.36(7.95-13.56)$ & $31.57(22.35-55.33)$ & $2.65 \pm 0.40$ & $2.122(4,0.713)$ & 1.8 & - \\
\hline \multirow[t]{4}{*}{ 4,5-dimethylfurfural } & D. farinae & $\begin{array}{l}\text { Contact }+ \text { fumigant } \\
\left(\mu \mathrm{g} / \mathrm{cm}^{2}\right)\end{array}$ & $9.95(8.60-11.46)$ & $16.30(13.62-23.48)$ & $5.97 \pm 1.22$ & $0.386(4,0.984)$ & 2.0 & - \\
\hline & D. pteronyssinus & $\begin{array}{l}\text { Contact }+ \text { fumigant } \\
\left(\mu \mathrm{g} / \mathrm{cm}^{2}\right)\end{array}$ & $9.91(8.43-11.61)$ & $17.87(14.56-26.53)$ & $5.00 \pm 0.96$ & $1.955(4,0.744)$ & 1.8 & - \\
\hline & H. longicornis & Packet (mg/ml) & $>50.00$ & - & - & - & - & - \\
\hline & T.putrescentiae & $\begin{array}{l}\text { Contact }+ \text { fumigant } \\
\left(\mu \mathrm{g} / \mathrm{cm}^{2}\right)\end{array}$ & $7.12(5.67-8.92)$ & $15.91(12.12-25.09)$ & $3.67 \pm 0.60$ & $1.187(4,0.880)$ & 2.6 & - \\
\hline \multirow[t]{4}{*}{ 5-hydroxymethylfurfural } & D. farinae & $\begin{array}{l}\text { Contact }+ \text { fumigant } \\
\left(\mu \mathrm{g} / \mathrm{cm}^{2}\right)\end{array}$ & $53.82(43.95-65.49)$ & $98.31(78.46-146.24)$ & $4.90 \pm 0.90$ & $1.226(4,0.874)$ & 0.4 & - \\
\hline & D. pteronyssinus & $\begin{array}{l}\text { Contact }+ \text { fumigant } \\
\left(\mu \mathrm{g} / \mathrm{cm}^{2}\right)\end{array}$ & $48.71(38.98-60.46)$ & 103.07 (79.73-158.01) & $3.94 \pm 0.65$ & $2.824(4,0.398)$ & 0.4 & - \\
\hline & H. longicornis & Packet (mg/ml) & $>50.00$ & - & - & - & - & - \\
\hline & T.putrescentiae & $\begin{array}{l}\text { Contact }+ \text { fumigant } \\
\left(\mu \mathrm{g} / \mathrm{cm}^{2}\right)\end{array}$ & $57.58(48.58-67.05)$ & $101.58(84.45-139.76)$ & $5.20 \pm 0.91$ & $4.058(4,0.398)$ & 0.3 & - \\
\hline \multirow[t]{4}{*}{ Amitraz } & D. farinae & $\begin{array}{l}\text { Contact }+ \text { fumigant } \\
\left(\mu \mathrm{g} / \mathrm{cm}^{2}\right)\end{array}$ & $-^{\mathrm{a}}$ & - & - & - & - & - \\
\hline & D. pteronyssinus & $\begin{array}{l}\text { Contact }+ \text { fumigant } \\
\left(\mu \mathrm{g} / \mathrm{cm}^{2}\right)\end{array}$ & - & - & - & - & - & - \\
\hline & H. longicornis & Packet (mg/ml) & $23.83(18.67-30.46)$ & $64.18(47.12-105.32)$ & $2.98 \pm 0.44$ & $3.379(4,0.497)$ & - & 1.0 \\
\hline & T.putrescentiae & $\begin{array}{l}\text { Contact }+ \text { fumigant } \\
\left(\mu \mathrm{g} / \mathrm{cm}^{2}\right)\end{array}$ & - & - & - & - & - & - \\
\hline \multirow[t]{4}{*}{ Benzyl benzoate } & D. farinae & $\begin{array}{l}\text { Contact }+ \text { fumigant } \\
\left(\mu \mathrm{g} / \mathrm{cm}^{2}\right)\end{array}$ & $12.11(10.49-14.03)$ & $22.94(18.86-31.76)$ & $4.62 \pm 0.71$ & $3.660(4,0.454)$ & 1.7 & - \\
\hline & D. pteronyssinus & $\begin{array}{l}\text { Contact }+ \text { fumigant } \\
\left(\mu \mathrm{g} / \mathrm{cm}^{2}\right)\end{array}$ & $11.09(9.62-12.77)$ & $20.45(17.01-27.64)$ & $4.83 \pm 0.73$ & $3.319(4,0.506)$ & 1.6 & - \\
\hline & H. longicornis & Packet $(\mathrm{mg} / \mathrm{ml})$ & $-^{\mathrm{a}}$ & - & - & - & - & - \\
\hline & T.putrescentiae & $\begin{array}{l}\text { Contact }+ \text { fumigant } \\
\left(\mu \mathrm{g} / \mathrm{cm}^{2}\right)\end{array}$ & $9.95(8.49-11.48)$ & $18.08(15.15-24.05)$ & $4.94 \pm 0.77$ & $1.016(4,0.907)$ & 1.9 & - \\
\hline \multirow[t]{4}{*}{ DEET } & D. farinae & $\begin{array}{l}\text { Contact }+ \text { fumigant } \\
\left(\mu \mathrm{g} / \mathrm{cm}^{2}\right)\end{array}$ & $20.26(17.12-24.49)$ & $44.14(34.22-68.35)$ & $3.79 \pm 0.59$ & $2.401(4,0.662)$ & 1.0 & - \\
\hline & D. pteronyssinus & $\begin{array}{l}\text { Contact }+ \text { fumigant } \\
\left(\mu \mathrm{g} / \mathrm{cm}^{2}\right)\end{array}$ & $17.40(15.12-20.31)$ & $31.77(25.98-45.00)$ & $4.90 \pm 0.79$ & $1.301(4,0.861)$ & 1.0 & - \\
\hline & H. longicornis & Packet (mg/ml) & - & - & - & - & - & - \\
\hline & T.putrescentiae & $\begin{array}{l}\text { Contact }+ \text { fumigant } \\
\left(\mu \mathrm{g} / \mathrm{cm}^{2}\right)\end{array}$ & $18.54(16.01-21.85)$ & $35.24(28.39-51.39)$ & $4.59 \pm 0.74$ & $2.226(4,0.694)$ & 1.0 & - \\
\hline
\end{tabular}



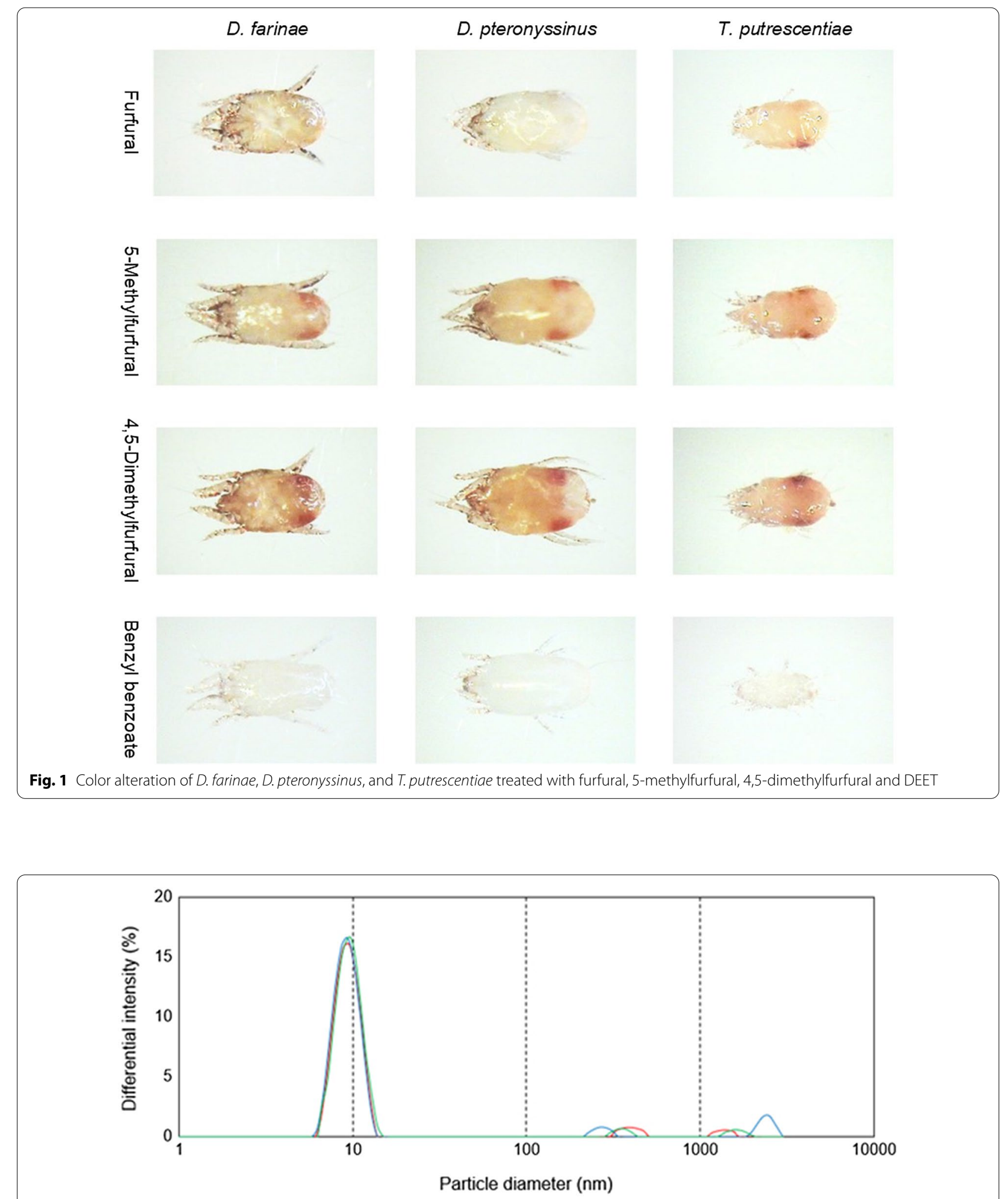

Fig. 2 Particle size distribution of 4,5-dimethylfurfural nanoemulsion. Mean droplet size, $161.3 \pm 32.3 \mathrm{~nm}$; Polydispersity index, $0.137 \pm 0.031$ 
Table 5 Acaricidal activities of 4,5-dimethylfurfural nanoemulsion against D. farinae, D. pteronyssinus and T. putrescentiae using spray bioassay

\begin{tabular}{|c|c|c|c|c|c|}
\hline Compound & Insect & $\mathrm{LD}_{50}\left(\mu \mathrm{g} / \mathrm{cm}^{2} ; 95 \% \mathrm{Cl}\right)$ & $\mathrm{LD}_{90}\left(\mu \mathrm{g} / \mathrm{cm}^{2} ; 95 \% \mathrm{Cl}\right)$ & Slope \pm SE & $\mathrm{X}^{2}(\mathrm{df}, p)$ \\
\hline \multirow[t]{3}{*}{ 4,5-dimethylfurfural nanoemulsion } & D. farinae & $7.24(5.84-8.71)$ & $15.96(12.80-22.50)$ & $3.74 \pm 0.57$ & $3.524(4,0.474)$ \\
\hline & D. pteronyssinus & $6.87(5.51-8.28)$ & $15.29(12.24-21.59)$ & $3.69 \pm 0.56$ & $3.860(4,0.425)$ \\
\hline & T.putrescentiae & $5.59(4.41-6.94)$ & 14.39 (10.97-22.09) & $3.12 \pm 0.47$ & $3.710(4,0.447)$ \\
\hline \multirow{3}{*}{$\begin{array}{l}\text { 4,5-dimethylfurfural emulsion dis- } \\
\text { solved in ethanol }\end{array}$} & D. farinae & $9.95(8.60-11.46)$ & $16.30(13.62-23.48)$ & $5.97 \pm 1.22$ & $0.386(4,0.984)$ \\
\hline & D. pteronyssinus & $9.91(8.43-11.61)$ & $17.87(14.56-26.53)$ & $5.00 \pm 0.96$ & $1.955(4,0.744)$ \\
\hline & T. putrescentiae & $7.12(5.67-8.92)$ & $15.91(12.12-25.09)$ & $3.67 \pm 0.60$ & $1.187(4,0.880)$ \\
\hline
\end{tabular}

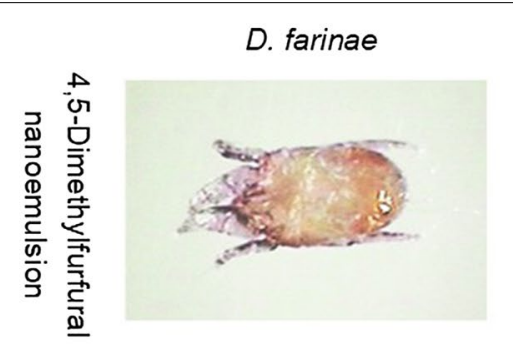

D. pteronyssinus
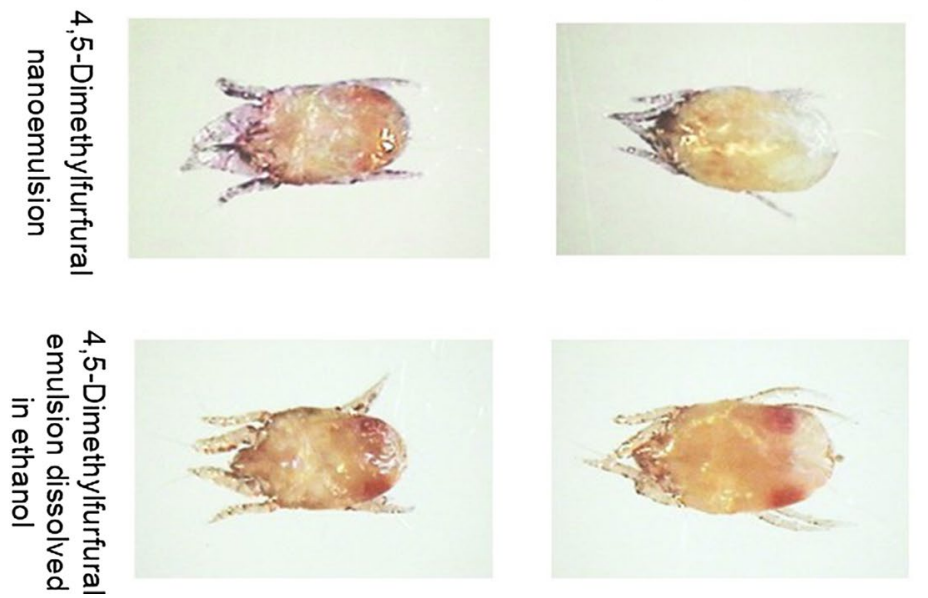

T. putrescentiae
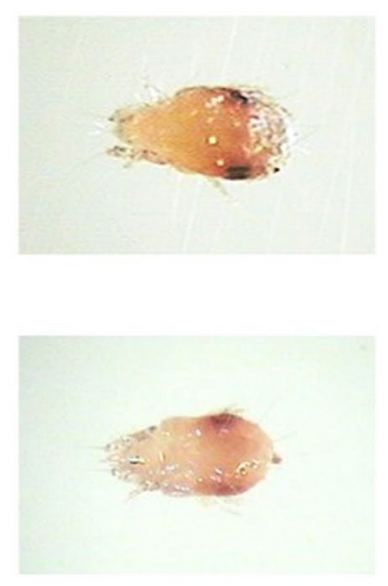

Fig. 3 Color alteration of D. farinae, D. pteronyssinus, and T. putrescentiae treated with 4,5-dimethylfurfural emulsion dissolved in ethanol and nanoemulsion

benzaldehyde influenced the acaricidal activity against $D$. farinae, D. pteronyssinus, and $H$. longicornis. These results seem to be attributed the difference in the existence of a methyl functional group.

One of the issues for practical uses of the essential oil or its plant-derived compound as commercial pesticides is its short lasting for acaricidal effect [17]. Furthermore, these plant-derived compounds are known to have poor water solubility. To overcome these problems, formulation into nanoemulsion has been developed. Nanoemulsion is also considered for enhancing water solubility and bioavailability of organic compounds. For these reasons, several studies trying to increase the bioactivities of nanoemulsions have been reported [32, 33]. This study also showed that the nanoemulsion of 4,5-dimethylfurfural, which was the most active compound among 5-methylfurfural derivatives, showed more effective acaricidal activity than the emulsion dissolved in ethanol against D. farinae, D. pteronyssinus, and T. putrescentiae. Regarding the mite color alteration, there was no difference between the ethanol-dissolved emulsion and nanoemulsion. 5-Methylfurfural and isovalerate from V. fauriei essential oil may suggest a new concept of acaricides due to the potential indicating feature by color alteration of synanthropic mites.

\section{Acknowledgements}

This research was supported by Government-wide R\&D Fund project for infectious disease research (GFID), Republic of Korea (Grant Number: HG18C0055).

\section{Authors' contributions}

S-AC carried out the experiments, prepared most of the data, and wrote the primary paper; JHP rewrote the paper; HSL proposed the key idea of this paper, designed the experiments, managed the research process, and wrote the paper; JHL managed the research process and rewrote the paper. All authors read and approved the final manuscript.

\section{Declarations}

\section{Competing interests}

The authors declare no competing interests.

\section{Author details}

${ }^{1}$ Department of Bioenvironmental Chemistry, College of Agriculture and Life Sciences, Jeonbuk National University, Jeonju 54896, Republic of Korea. ${ }^{2}$ Biomedical Research Team, HS Biotech \& Holdings (HSBH) for Medical Research, 
Basel, Switzerland. ${ }^{3}$ Department of Agricultural Convergence Technology, Jeonbuk National University, Jeonju 54896, Republic of Korea.

Received: 27 October 2021 Accepted: 19 December 2021 Published online: 29 December 2021

\section{References}

1. Cuthbert OD, Brostoff J, Wraith DG, Brightons WD (1979) 'Barn allergy': asthma and rhinitis due to storage mites. Clin Exp Allergy 9:229-236

2. Solarz K, Senczuk L, Maniurka H, Cichecka E, Peszke M (2007) Comparisons of the allergenic mite prevalence in dwellings and certain outdoor environments of the Upper Silesia (southwest Poland). Int J Hyg Environ Health 210:715-724

3. Sinha RN, Wallace HAH (1966) Association of granary mites and seedborne fungi in stored grain and in outdoor and indoor habitats. Ann Entomol Soc Am 59:1170-1181

4. Zhendong H, Guangfu Y, Zhong Z, Ruiling Z (2019) Phylogenetic relationships and effectiveness of four Beauveria bassiana sensu lato strains for control of Haemaphysalis longicornis (Acari: Ixodidae). Exp Appl Acarol 77:83-92

5. Ding F, Zhang W, Wang L, Hu W, Soares Magalhaes RJ, Sun H, Zhou H, Sha S, Li S, Liu Q, Li Q, Yang W, Huang L, Li C, Yin W (2013) Epidemiologic features of severe fever with thrombocytopenia syndrome in China. Clin Infect Dis 56:1682-1683

6. Gai ZT, Zhang Y, Liang MF, Jin C, Zhang S, Zhu CB, Li C, Li XY, Zhang QF, Bian PF, Zhang LH, Wang B, Zhou N, Liu JX, Song XG, Xu A, Bi ZQ, Chen SJ, Li DX (2012) Clinical progress and risk factors for death in severe fever with thrombocytopenia syndrome patients. J Infect Dis 206:1095-1102

7. Higa LOS, Garcia MV, Barros JC, Koller WW, Andreotti R (2015) Acaricide resistance status of the Rhipicephalus microplus in Brazil: a literature overview. Med chem 5:326-333

8. Isman MB (2005) Botanical insecticides, deterrents, and repellents in modern agriculture and an increasingly regulated world. Annu Rev Entomol 51:45-66

9. Fraenkel GS (1959) The raison d'etre of secondary plant substances. Science 129:1466-1470

10. Chen HW, Wei BJ, He XH, Liu Y, Wang J (2015) Chemical components and cardiovascular activities of Valeriana spp. Evid-based Complement Altern Med. https://doi.org/10.1155/2015/947619

11. Dua V, Alam M, Pandey A, Rai S, Chopra A, Kaul V, Dash A (2008) Insecticidal activity of Valeriana jatamansi (Valerianaceae) against mosquitoes. J Am Mosq Control Assoc 24:315-318

12. Kim J, Seo SM, Lee SG, Shin SC, Park IK (2008) Nematicidal activity of plant essential oils and components from coriander (Coriandrum sativum), oriental sweetgum (Liquidambar orientalis), and valerian (Valeriana wallichii) essential oils against pine wood nematode (Bursaphelenchus xylophilus). J Agric Food Chem 56:7316-7320

13. Gupta A, Eral HB, Hatton TA, Doyle PS (2016) Nanoemulsions: formation, properties and applications. Soft Matter 12:2826-2841

14. Park JH, Lee HS (2018) Acaricidal target and mite indicator as color alteration using 3,7-dimethyl-2,6-octadienal and its derivatives derived from Melissa officinalis leaves. Sci Rep 8:8129

15. Lee MJ, Lee SE, Kang MS, Park B, Lee SG, Lee HS (2018) Acaricidal and insecticidal properties of Coriandrum sativum oils and their major constituents extracted by three different methods against stored product pests. Appl Biol Chem 61:481-488

16. Adams RP (2007) Identification of essential oil components by gas chromatography/mass spectrometry, 4th edn. Allured Pub Corp, Carol Stream

17. Sugumar S, Clarke SK, Nirmala MJ, Tyagi BK, Mukherjee A, Chandrasekaran N (2014) Nanoemulsion of eucalyptus oil and its larvicidal activity against Culex quinquefasciatus. Bull Entomol Res 104:393-402

18. Godara R, Katoch R, Yadav A, Ahanger RR, Bhutyal ADS, Verma PK, Katoch M, Dutta S, Nisa F, Singh NK (2015) In vitro acaricidal activity of ethanolic and aqueous floral extracts of Calendula officinalis against synthetic pyrethroid resistant Rhipicephalus (Boophilus) microplus. Exp Appl Acarol 67:147-157

19. Abbott WS (1925) A method of computing the effectiveness of an insecticide. J econ Entomol 18:265-267
20. Song JE, Lee HS (2018) Mite color alteration and acaricidal activity of 3,7-dimethyl-2,6-octadienal and its structural analogues against the stored food pest mite Tyrophagus putrescentiae. Exp Appl Acarol 76:355-363

21. Kang MS, Lee HS (2018) Acaricidal and insecticidal responses of Cinnamomum cassia oils and main constituents. Appl Biol Chem 61:653-659

22. Kwon JH, Ahn YJ (2002) Acaricidal activity of butylidenephthalide identified in Cnidium officinale rhizome against Dermatophagoides farinae and Dermatophagoides pteronyssinus (acari: Pyroglyphidae). J Agric Food Chem 50:4479-4483

23. Pavlovic M, Kovacevic N, Tzakou O, Couladis M (2004) The essential oil of Valeriana officinalis L. s.l. growing wild in western Serbia. J Essent Oil Res 16:397-399

24. Javidnia K, Miri R, Kamalinejad M, Khazraii H (2006) Chemical composition of the volatile oil of aerial parts of Valeriana sisymbriifolia Vahl. grown in Iran. Flavour Frag J 21:516-518

25. Wang J, Zhao J, Liu H, Zhou L, Liu Z, Wang J, Han J, Yu Z, Yang F (2010) Chemical analysis and biological activity of the essential oils of two valerianaceous species from China: Nardostachys chinensis and Valeriana officinalis. Molecules 15:6411-6422

26. Liu XC, Zhou L, Liu ZL (2013) Identification of insecticidal constituents from the essential oil of Valeriana jatamansi Jones against Liposcelis bostrychophila Badonnel. J Chem. https://doi.org/10.1155/2013/853912

27. Odonbayar B, Murata T, Batkhuu J, Yasunaga K, Goto R, Sasaki K (2016) Antioxidant flavonols and phenolic compounds from Atraphaxis frutescens and their inhibitory activities against insect phenoloxidase and mushroom tyrosinase. J Nat Prod 79:3065-3071

28. Sugumaran M (2009) Complexities of cuticular pigmentation in insects. Pigment Cell Melanoma Res 22:523-525

29. Lee $\mathrm{CH}$, Lee HS (2008) Acaricidal activity and function of mite indicator using plumbagin and its derivatives isolated from Diospyros kaki Thunb. roots (Ebenaceae). J Microbiol Biotechnol 18:314-321

30. Yang JY, Kim MG, Park JH, Hong ST, Lee HS (2014) Evaluation of benzaldehyde derivatives from Morinda officinalis as anti-mite agents with dual function as acaricide and mite indicator. Sci Rep 4:7149-7145

31. Oh MS, Yang JY, Lee HS (2012) Acaricidal toxicity of 2'-hydroxy-4'methylacetophenone isolated from Angelicae koreana roots and structure-activity relationships of its derivatives. J Agric Food Chem 60:3606-3611

32. Balasubramani S, Rajendhiran T, Moola AK, Diana RKB (2017) Development of nanoemulsion from Vitex negundo L. essential oil and their efficacy of antioxidant, antimicrobial and larvicidal activities (Aedes aegypti L.). Environ Sci Pollut Res 24:15125-15133

33. Oliveira AEMFM, Duarte JL, Cruz RAS, Souto RNP, Ferreira RMA, Peniche T, da Conceição EC, de Oliveira LAR, Faustino SMM, Florentino AC, Carvalho $J C T$, ernandes CPF, (2017) Pterodon emarginatus oleoresin-based nanoemulsion as a promising tool for Culex quinquefasciatus (Diptera: Culicidae) control. J Nanobiotechnol 15:1-11

\section{Publisher's Note}

Springer Nature remains neutral with regard to jurisdictional claims in published maps and institutional affiliations. 